\title{
Adult Primary Peripheral PNET/Ewing's Sarcoma of the Cervical and Thoracic Spine
}

\author{
RAAMIS KHWAJA ${ }^{1}$, EMMANUEL MANTILLA ${ }^{1}$, KAREN FINK $^{2}$ and EDWARD PAN ${ }^{1}$ \\ ${ }^{1}$ UT Southwestern Medical Center, Dallas, TX, U.S.A.; \\ ${ }^{2}$ Baylor University Medical Center, Dallas, TX, U.S.A.
}

\begin{abstract}
This case report describes a patient with a rare occurrence of primary spinal intramedullary Ewing's sarcoma (ES) in the cervical and thoracic spine. The older age of disease occurrence, uncommon location in the cervical and thoracic spine, and EWSRI gene fusion as the basis of diagnosis are unique features of this case. There is no clear protocol for treatment of primary extraskeletal ES of the spine, with controversy between evidence for pursuing surgery versus a combination of radiation and chemotherapy. Our patient was treated with temozolomide chemotherapy for recurrent metastatic disease of primary ES of the spine.
\end{abstract}

\section{Case Report}

A 44-year-old female patient initially presented in 2009 with shooting pain in her right upper extremity, which gradually progressed to involve her left upper extremity and bilateral lower extremities (BLE), leading to paraplegia and loss of sensation below the chest in the span of a few months. Spine magnetic resonance imaging (MRI) with contrast demonstrated an intramedullary enhancing lesion in the C7-T1 region, with multiple enhancing nodules distributed throughout the spine consistent with leptomeningeal dissemination. The patient underwent sub-total resection of the neurologically symptomatic cervical lesion followed by cranio-spinal irradiation (CSI) and chemotherapy involving the 'Packer regimen,' consisting of 6 cycles of cisplatin and lomustine. Pathology review of her tumor demonstrated a primary undifferentiated neuroendocrine carcinoma. Next generation sequencing (NGS) by Foundation One (Copyright) detected genomic alterations in the PIK3 pathway (PIK3CA Q546R - subclonal) and EWSR1-FLII fusion (IGF-1 mutation). Her enhancing lesions disappeared

Correspondence to: Edward Pan, MD, UT Southwestern Medical Center, 5323 Harry Hines Blvd. ND3.300A Dallas, TX 75390-9186, U.S.A. Tel: +1 2146458186, e-mail: Edward.pan@utsouthwestern.edu

Key Words: Ewing's Sarcoma, CNS, primitive neuroectodermal tumor, gene fusion, immunohistochemistry, chemotherapy, spinal. after the initial treatment regimen. Follow-up imaging in August 2012 revealed a discrete recurrent lumbar metastasis behind the L4 vertebra, and she received Cyberknife stereotactic radiosurgery (SRS) followed by ICE (ifosfamide, carboplatin, etoposide) chemotherapy for 5 of 6 planned cycles; due to chemotherapy side effects she did not complete the 6th cycle. The patient was stable and apparently disease-free until August 2016, when she presented with worsening gait, multiple falls, and BLE weakness. MRI of the spine showed recurrence of another discrete lumbar metastasis behind L2, which was treated again with Cyberknife SRS. She developed multiple leptomeningeal recurrent tumor nodules in July of 2017, and was treated with 3 months of Afinitor $10 \mathrm{mg}$ daily through December 2017. She progressed on Afinitor and was treated with 8 cycles of monthly temozolomide at $200 \mathrm{mg} / \mathrm{m}^{2} / \mathrm{day} \times 5$ every 28 days. She had progression again in August of 2018, and is receiving carboplatin at an AUC of 4 every 4 weeks with bevacizumab $10 \mathrm{mg} / \mathrm{kg}$ every 2 weeks. She is tolerating this treatment regimen well and had a partial tumor response. She has received 7 cycles to date and is currently radiographically and clinically stable.

\section{Discussion}

The Ewing's sarcoma family of tumors (ESFT) is most commonly comprised of primary Ewing's sarcoma (ES) of the bone, but also includes extraskeletal ES, peripheral primitive neuroectodermal tumor (pPNET), and Askin tumor (thoracopulmonary PNET) (1). ES is primarily a childhood and adolescent malignancy, with peak incidence in the second decade of life (2), and belongs to the subset of PNETs (3). While the majority of ESFT are primary bone tumors, soft tissue can also be involved, most commonly at the paravertebral region and less so at the epidural space (4). In contrast, intradural involvement by CNS-PNETs (Central nervous system PNET) is exceedingly rare (5).

From 1978-2011, there have been 29 reported cases of primary spinal intramedullary CNS-PNETs across 22 case reports (6-8). Among these 29 cases, the average age of occurrence of primary spinal ES was 19 (ranges from ages 
6-32) (8). Our patient's age of 44 years old at time of tumor diagnosis falls considerably outside the upper end of this range.

Ewing's sarcoma and related pPNETs have been demonstrated to share a recurrent chromosomal translocation, $\mathrm{t}(11 ; 22)(\mathrm{q} 24 ; \mathrm{q} 12)$, which fuses a portion of the EWSR1 gene on chromosome 22 to a portion of the FLII gene on chromosome 11 (9). The EWS gene codes for a highly conserved RNA binding protein containing an RNA recognition motif, allowing for interaction with RNA or single-stranded DNA (10). The FLII gene codes for the FLI protein, which belongs to the ETS (erythroblastosis virustransforming sequence) oncogene family and mediates cellular development and oncogenesis $(11,12)$. The ETS domain, constituted by a highly conserved 85 -amino acid domain, allows for specific binding to DNA sequences (13). As a result of the EWSRI-FLII gene translocation, the expressed fusion protein is able to bind DNA via the ETS domain and control gene expression via the RNA-binding properties of the EWS protein. Approximately $85-90 \%$ of ESFTs are characterized by this $\mathrm{t}(11 ; 22)$ ESWR1-FLI1 translocation (14).

Our patients' tumor had genomic alterations in the PIK3CA pathway as well as IGF-1 with an EWSRI-FLII fusion. There has been one other case documented to have an ESWRI gene rearrangement as the histopathologic foundation for the diagnosis of primary epidural ES (15). The reported incidence of primary intraspinal ES or pPNET is twice as high in the lumbar region as the cervical or thoracic region, making the location of this tumor in the C7T1 region even more uncommon (8). Our case is the first reported female patient with cervical and thoracic involvement with the diagnosis confirmed by the presence of the ESWRI gene fusion using immunohistochemistry.

Due to the limited number of reported patient cases, primary ES affecting the spine has no definite treatment protocol, though en bloc resection with safe margins, if possible, is currently regarded as the most important initial prognostic intervention $(15,16)$. Kinsella et al. have reported that the combination of chemotherapy and radiotherapy is more important for management than surgery, especially given the difficulty of achieving a gross total resection in these patients $(17,18)$. The decision to pursue surgery, even in cases of spinal cord compression from ES, is controversial. Mirzaei et al. performed a retrospective study of fifteen patients with primary ES of the spine and found that chemotherapy can be effective as an initial treatment option even in cases of severe spinal cord compression (19).

\section{Conclusion}

In conclusion, to the best of our knowledge, this is the only reported case of recurrent metastatic primary ES of the cervical spine, where temozolomide was used as part of the treatment. Although the patient subsequently progressed, she was able to achieve at least eight months of progression-free survival on temozolomide. All such rare tumors that involve the CNS should be analyzed by NGS in order to better characterize and understand their behavior and ultimately determine their most effective treatments.

\section{Conflicts of Interest}

Dr. Emmanuel Mantilla's fellowship was partially funded by an educational grant from AbbVie pharmaceuticals. There are no other conflicts of interest to report.

\section{Authors' Contributions}

Raamis Khwaja: Primary author of manuscript, helped conduct literature review and wrote the abstract, case report, discussion, and conclusions sections of the paper; Emmanuel Mantilla: Helped conduct literature review, wrote and edited portions of case report, discussion and conclusion sections of paper; Karen Fink: Contributed clinical data, reviewed and edited case report and discussion; Edward Pan: Senior author of manuscript, contributed clinical data, edited the case report, discussion and conclusion sections of paper, oversaw research conduct.

\section{Acknowledgements}

Dr. Emmanuel Mantilla's fellowship was partially funded by an educational grant from AbbVie pharmaceuticals.

\section{References}

1 Javery O, Krajewski K, O'Regan K, Kis B, Giardino A, Jagannathan J and Ramaiya NH: A to $\mathrm{z}$ of extraskeletal ewing sarcoma family of tumors in adults: Imaging features of primary disease, metastatic patterns, and treatment responses. AJR Am J Roentgenol 197(6): W1015-1022, 2011. PMID: 22109315. DOI: 10.2214/AJR.11.6667

2 Dogan S, Lekovic GP, Theodore N, Horn EM, Eschbacher J and Rekate HL: Primary thoracolumbar ewing's sarcoma presenting as isolated epidural mass. Spine J 9(1): e9-14, 2009. PMID: 18201936. DOI: 10.1016/j.spinee.2007.11.003

3 Shoubash L, Nowak S, Vogelgesang S, Schroeder HWS and Muller JU: Surgical management of an adult manifestation of ewing sarcoma of the spine-a case report. AME Case Rep 2: 34, 2018. PMID: 30264030. DOI: 10.21037/acr.2018.06.07

4 Eggli KD, Quiogue T and Moser RP Jr.: Ewing's sarcoma. Radiol Clin North Am 31(2): 325-337, 1993. PMID: 8446752

5 Lozupone E, Martucci M, Rigante L, Gaudino S, Di Lella GM and Colosimo C: Magnetic resonance image findings of primary intradural ewing sarcoma of the cauda equina: Case report and review of the literature. Spine J 14(4): e7-e11, 2014. PMID: 24314762. DOI: 10.1016/j.spinee.2013.09.024.

6 Kampman WA, Kros JM, De Jong TH and Lequin MH: Primitive neuroectodermal tumours (pnets) located in the spinal canal; the relevance of classification as central or peripheral pnet: Case report of a primary spinal pnet occurrence with a critical literature review. J Neurooncol 77(1): 65-72, 2006. PMID: 16292490. DOI: 10.1007/s11060-005-9006-Z 
7 Kumar R, Reddy SJ, Wani AA and Pal L: Primary spinal primitive neuroectodermal tumor: Case series and review of the literature. Pediatr Neurosurg 43(1): 1-6, 2007. PMID: 17190980. DOI: $10.1159 / 000097517$

8 Saeedinia S, Nouri M, Alimohammadi M, Moradi $\mathrm{H}$ and Amirjamshidi A: Primary spinal extradural ewing's sarcoma (primitive neuroectodermal tumor): Report of a case and metaanalysis of the reported cases in the literature. Surg Neurol Int 3: 55, 2012. PMID: 22629492. DOI: 10.4103/2152-7806.96154

9 Delattre O, Zucman J, Plougastel B, Desmaze C, Melot T, Peter M, Kovar H, Joubert I, de Jong P, Rouleau G et al: Gene fusion with an ets DNA-binding domain caused by chromosome translocation in human tumours. Nature 359(6391): 162-165, 1992. PMID: 1522903 . DOI: $10.1038 / 359162 \mathrm{a} 0$

10 Ohno T, Ouchida M, Lee L, Gatalica Z, Rao VN and Reddy ES: The ews gene, involved in ewing family of tumors, malignant melanoma of soft parts and desmoplastic small round cell tumors, codes for an RNA binding protein with novel regulatory domains. Oncogene 9(10): 3087-3097, 1994. PMID: 8084618.

11 Zucman J, Delattre O, Desmaze C, Plougastel B, Joubert I, Melot T, Peter M, De Jong P, Rouleau G, Aurias A and et al.: Cloning and characterization of the ewing's sarcoma and peripheral neuroepithelioma $\mathrm{t}(11 ; 22)$ translocation breakpoints. Genes Chromosomes Cancer 5(4): 271-277, 1992. PMID: 1283315.

12 Wasylyk B, Hahn SL and Giovane A: The ets family of transcription factors. Eur J Biochem 211(1-2): 7-18, 1993. PMID: 8425553.

13 Burd CG and Dreyfuss G: Conserved structures and diversity of functions of rna-binding proteins. Science 265(5172): 615-621, 1994. PMID: 8036511.

14 Hromas $\mathrm{R}$ and Klemsz M: The ets oncogene family in development, proliferation and neoplasia. Int J Hematol 59(4): 257-265, 1994. PMID: 8086619.
15 Giner J, Isla A, Cubedo R and Tejerina E: Primary epidural lumbar ewing sarcoma: Case report and review of the literature. Spine (Phila Pa 1976) 41(6): E375-378, 2016. PMID: 26966980. DOI: $10.1097 / \mathrm{BRS} .0000000000001246$

16 Zhang J, Huang Y, Lu J, He A, Zhou Y, Hu H, Shen Z, Sun Y and Yao Y: Impact of first-line treatment on outcomes of ewing sarcoma of the spine. Am J Cancer Res 8(7): 1262-1272, 2018. PMID: 30094099.

17 Kinsella TJ, Triche TJ, Dickman PS, Costa J, Tepper JE and Glaubiger D: Extraskeletal ewing's sarcoma: Results of combined modality treatment. J Clin Oncol 1(8): 489-495, 1983. PMID: 6668512. DOI: 10.1200/JCO.1983.1.8.489

18 Subbiah V, Anderson P, Lazar AJ, Burdett E, Raymond K and Ludwig JA: Ewing's sarcoma: Standard and experimental treatment options. Curr Treat Options Oncol 10(1-2): 126-140, 2009. PMID: 19533369. DOI: 10.1007/s11864-009-0104-6

19 Mirzaei L, Kaal SE, Schreuder HW and Bartels RH: The neurological compromised spine due to ewing sarcoma. What first: Surgery or chemotherapy? Therapy, survival, and neurological outcome of 15 cases with primary ewing sarcoma of the vertebral column. Neurosurgery 77(5): 718-724; discussion 724-715, 2015. PMID: 26308634. DOI: 10.1227/ NEU.0000000000000903
Received May 24, 2019

Revised June 15, 2019

Accepted June 18, 2019 